\title{
Ensino de Redes de Computadores Mediado por Tecnologias Educacionais: um Mapeamento Sistemático da Literatura
}

\author{
Alisson Emanuel Diniz Santos - IFMA/Campus São João dos Patos - \\ alissondinizsantos@gmail.com
}

Thiago Reis da Silva - IFMA/Campus São João dos Patos - thiago.reis@ifma.edu.br

Felipe Gonçalves dos Santos - IFPI/Campus Corrente - felipe.santos@ifpi.edu.br

Filipe Fontinele de Almeida - FAESF/PI - filipefontineli@gmail.com

Julian Rodrigues Valério - IFPI/Campus São Raimundo Nonato -

julian.valerio@ifpi.edu.br

Eduardo Henrique da Silva Aranha - UFRN/PPgSC - eduardoaranha@dimap.ufrn.br

Resumo. O ensino na área de Redes de Computadores, assim como em outras áreas da computação é de difícil entendimento para grande parte dos estudantes, sendo assim um desafio para os professores da área. Por outro lado, novas abordagens tecnológicas investigadas pela academia muitas vezes não chegam ao conhecimento dos professores $e$, consequentemente, às salas de aula. Visando levar este conhecimento a sociedade, este artigo apresenta os resultados de um Mapeamento Sistemático da Literatura (MSL) que buscou investigar e identificar, em nivel nacional, as principais tecnologias educacionais e estratégias pedagógicas utilizadas como mediadoras para o ensino de redes de computadores. Após a seleção dos estudos primários, foram analisados 36 estudos, os quais indicaram que Ferramentas e Jogos são as abordagens mais utilizadas. Os resultados ainda apresentam a baixa aplicação de métodos empíricos como meio de avaliação dos resultados obtidos pelas pesquisas desenvolvidas.

Palavras-chave: Redes de Computadores, Tecnologias Educacionais, Mapeamento Sistemático da Literatura.

\section{Teaching of Computer Networks Mediated by Educational Technologies: A Systematic Mapping of Literature}

\begin{abstract}
Teaching in the area of Computer Networks, as well as in other areas of computing, is difficult for most students to understand, thus being a challenge for teachers in the area. On the other hand, new technological approaches investigated by the academy often do not reach the knowledge of the teachers and, consequently, the classrooms. In order to bring this knowledge to society, this article presents the results of a Systematic Mapping of Literature (MSL) that sought to investigate and identify, at the national level, the main educational technologies and pedagogical strategies used as mediators for the teaching of computer networks. After selecting the primary studies, 36 studies were analyzed, which indicated that Tools and Games are the most used approaches. The results still show the low application of empirical methods as a means of evaluating the results obtained by the research developed.
\end{abstract}

Keywords: Computer Networks, Educational Technologies, Systematic Mapping of Literature.

\section{Introdução}

Ao falar das disciplinas da área de computação é comum ouvir relatos que os conteúdos são de difícil entendimento, levando em consideração que muitos conceitos são "abstratos" e, sendo assim, desvinculados com a realidade e o cotidiano de quem os estuda, causam certas barreiras entre os alunos e as disciplinas referentes à área. Em se 
tratando de Redes de Computadores (RC), as dificuldades são similares, principalmente em assuntos como as camadas dos modelos arquiteturais, as topologias, a lógica de endereçamento IP (Internet Protocol) ou segurança de redes.

Sendo assim, o desenvolvimento de atividades práticas em disciplinas de RC é essencial para o desenvolvimento de habilidades técnicas fundamentais dos estudantes. Muitas vezes os alunos não contam com um espaço físico completo que suporte a execução das práticas exigidas pela disciplina (FERREIRA et. al., 2014). Hassan (2003) destaca que em relação ao ensino na área de $\mathrm{RC}$, os problemas e dificuldades encontrados para o desenvolvimento da disciplina são: material didático e pouca disponibilidade de equipamentos adequados para a utilização de ferramentas. Para Costa, Viana e Coutinho (2017) isto ocorre em grande parte devido à velocidade em que os avanços tecnológicos acontecem e ao custo de manutenção de um laboratório experimental.

Dentro deste contexto, considerando essas observações, a utilização de jogos, simuladores, laboratórios virtuais, ferramentas, entre outras metodologias (computação desplugada e objetos de aprendizagem) acabam se tornando uma prática para minimizar essas dificuldades. Por essas razões, este trabalho investiga a literatura brasileira sobre a utilização de abordagens tecnológicas - jogos, simuladores, ferramentas, laboratórios virtuais, objetos de aprendizagem - para o ensino e aprendizagem de RC como auxílio no processo educacional. Para esse fim e almejando o alcance de resultados de valor científico, optou-se pelo Mapeamento Sistemático da Literatura (MSL).

Para fundamentar novas pesquisas que insiram de forma efetiva o ensino de $\mathrm{RC}$ com o uso de diversos recursos tecnológicos, é importante o conhecimento dos trabalhos relacionados existentes e das experiências reportadas que considerem as características e desafios encontrados no cenário do ensino nacional. Por exemplo, é importante se observar quais abordagens vêm sendo propostas e para qual público alvo, bem como os resultados obtidos e limitações observadas. Essa informação ajuda não só aos pesquisadores a identificarem questões de pesquisa em aberto, como também aos professores selecionarem tecnologias educacionais modernas propostas pela academia.

Para reportar o trabalho realizado, as demais seções deste artigo encontram-se organizadas da seguinte forma. Na Seção 2 é apresentado o planejamento do MSL, as questões de pesquisa, o processo de busca, os critérios de inclusão e exclusão e a coleta e análise dos dados dos artigos. Já a Seção 3 discute as questões de pesquisa de acordo com os resultados encontrados, as limitações do estudo e os trabalhos relacionados. Por fim, na Seção 4 são descritas as considerações finais.

\section{Planejamento do Mapeamento Sistemático}

Segundo Kitchenham et al. (2009), uma Revisão Sistemática da Literatura (RSL) deve ser realizada seguindo as etapas de planejamento, condução e documentação da revisão, podendo essas etapas serem também utilizadas no contexto de um MSL. Esta seção apresenta o planejamento do MSL realizado, conforme o modelo proposto por Kitchenham et al. (2009), visando responder às questões apresentadas na Tabela 1.

\section{Tabela 1: Questões de Pesquisa (QP).}

\begin{tabular}{l|l|}
\hline QPs & \multicolumn{1}{|c|}{ Descrição } \\
\hline QP1 & $\begin{array}{l}\text { Quais as abordagens (jogos, ferramentas, simulações, laboratórios virtuais, metodologias e } \\
\text { outras) estão sendo propostas para o ensino de Redes de Computadores? }\end{array}$ \\
\hline QP2 & Que conteúdos estão sendo abordados nos estudos? \\
\hline QP3 & Quais concepções e/ou teorias pedagógicas estão sendo utilizadas nos estudos? \\
\hline QP4 & Os estudos desenvolvidos suportam algum tipo de Tecnologia Assistiva? \\
\hline QP5 & Como está sendo realizada a avaliação dos estudos? \\
V.18 $\mathrm{N}^{\circ} 1$, julho, 2020_RENE \\
DOI:
\end{tabular}


Para Kitchenham et. al. (2009), alguns critérios devem ser atendidos para orientar uma seleção adequada dos estudos primários a serem analisados no mapeamento, a saber: população, intervenção e resultados. A população refere-se ao grupo populacional que será observado no MSL. Neste estudo, a população consiste em publicações (artigos completos, relatos de experiência, entre outros) com enfoque no "ensino de Redes de Computadores". A intervenção refere-se ao que será observado no contexto do MSL. Neste caso, são observados, por exemplo, os tipos de abordagens empregadas no ensino de RC. Os resultados referem-se aos achados esperados ao final do MSL. Neste caso, como resultados esperados, têm-se: i) a catalogação e classificação bibliográfica dos trabalhos que abordam ensino de RC; ii) a totalização dos tipos de abordagens utilizadas no ensino de $\mathrm{RC}$; iii) o levantamento das teorias pedagógicas utilizadas para desenvolvimento das abordagens; e iv) o levantamento de como está sendo realizada as avaliações dos estudos.

\subsection{Processo de busca}

O processo de busca de estudos primários consistiu em: i) utilizar o Google Scholar no processo de busca automática; ii) realizar buscas manuais nos anais de eventos nacionais relacionadas ao tema, a saber: Escola Regional de Redes de Computadores (ERRC), Computer and Beach (COTB), Congresso Internacional de Informática Educativa (TISE); Encontro Nacional de Computação dos Institutos Federais (ENCompIF), Simpósio Brasileiro de Informática na Educação (SBIE), Simpósio Brasileiro de Jogos e Entretenimento Digital (SBGAMES), Simpósio Brasileiro de Redes de Computadores e Sistemas Distribuidos (SBRC), Simpósio Brasileiro de Sistemas de Informação (SBSI), Workshop do Congresso Brasileiro de Informática na Educação (WCBIE), Workshop de Desafios da Computação Aplicada à Educação (DesafIE), Workshop de Educação em Computação (WEI) e Workshop de Informática na Escola (WIE); e iii) realizar buscas no site das revistas: Informática na Educação: Teoria \& Prática (IETP), Revista Brasileiro de Informática na Educação (RBIE), Revista Brasileira de Computação Aplicada (RBCA), Revista de Informática Teórica e Aplicada (RITA) e Revista Novas Tecnologias na Educação (RENOTE), utilizando o mecanismo de busca próprio destes sites; e iv) aplicar a técnica de backward snowballing "bola de neve" de forma interativa. Esta técnica identifica possíveis estudos primários relevantes em suas referências (JALALI e WOHLIN, 2012).

A busca automática foi realizada através de uma string de busca com os termos principais de interesse, derivados das QPs (KITCHENHAM et al., 2009). A string foi construída usando os operadores lógicos AND e OR, como mostrado a seguir:

\section{("educação" OR "ensino" OR “aprendizagem” OR "jogo” OR "sério") AND ("jogo" OR "simulação" OR "objeto de aprendizagem" OR "simuladores" OR "laboratório virtual” OR "software" OR "ferramenta”) AND ("redes de computadores”)}

Considerando o objetivo da pesquisa envolvendo diversos domínios, como, informática na educação e redes de computadores, selecionamos como ferramenta de busca o Google Scholar. A opção pelo Google Scholar justifica-se porque os artigos publicados em revistas reconhecidas cientificamente são encontrados por esta ferramenta, mas também permite a busca de artigos publicados em outras fontes de pesquisas como bibliotecas digitais ou repositórios digitais, com isso tornam-se possível a busca em diversos meios e domínios de publicação on-line. Analisamos apenas as 13 primeiras páginas dos resultados do Google Scholar, pois a partir da décima terceira página os artigos apresentados não possuíam relação com o tema de pesquisa.

\subsection{Critérios de Inclusão e Exclusão}


A análise dos artigos encontrados pela busca manual e automática foi realizada em quatro etapas. $\mathrm{Na}$ primeira etapa foram realizadas buscas utilizando a busca manual e a string de busca. Esta etapa filtrou os artigos analisando-se o título e data de publicação, mantendo apenas os que satisfizessem os seguintes Critérios de Inclusão (CI):

- CI1: Estudos que relataram o uso de alguma abordagem no ensino e aprendizagem de RC, em qualquer nível de ensino (ex., médio ou superior);

- CI2: Artigos publicados entre 1 de janeiro de 2014 a 31 de dezembro de 2019.

Nas três etapas seguintes, foram realizadas a leitura e análise do resumo (etapa 2), seguida da introdução e conclusão (etapa 3) e, por fim, a leitura completa dos artigos (etapa 4). Além de verificar novamente os critérios de inclusão, foram excluídos trabalhos que atendessem aos seguintes Critérios de Exclusão (CE):

- CE1: Estudos cujo foco principal não foi sobre o uso das tecnologias educacionais para o ensino e aprendizagem de RC;

- CE2: Documentos que estão disponíveis na forma de resumos, capítulos de livros ou apresentações e estudos secundários (ou seja, RSL e MSL);

- CE3: Trabalhos cujo texto completo não esteja disponível para acesso gratuitamente na $\mathrm{Web}$;

- CE4: Artigos duplicados.

É importante também ressaltar que, em alguns casos, a leitura de outras partes dos artigos foi realizada de maneira antecipada para aumentar o grau de confiança da seleção. Além disso, estudos semelhantes que apresentavam a evolução de uma mesma pesquisa e que continham os mesmos autores tiveram suas versões mais antigas removidas para se evitar duplicações (CE4).

\subsection{Procedimentos executados}

Para a realização deste MSL foi realizado o seguinte procedimento metodológico, com a participação de quatro pesquisadores com experiência no tema: 1) Aplicou-se o método de pesquisa para identificação dos potenciais estudos primários relacionados ao tema deste MSL, através da busca manual e string de pesquisa; 2) Para identificação dos estudos primários preliminares, um pesquisador realizou a leitura do título, do resumo e das palavras-chave da publicação, aplicando os critérios (CI e CE) descritos na Subseção 2.2; 3) Tendo como resultado um conjunto preliminar de estudos, este mesmo pesquisador realizou a divisão dos artigos para leitura entre os pesquisadores; 4) Os resultados obtidos após o item (2) foram então revisados pelos outros pesquisadores envolvidos neste estudo e eventuais desacordos foram discutidos e solucionados, por meio da manutenção ou da remoção do trabalho previamente selecionado pelo primeiro pesquisador; 5) Tendo como resultado o conjunto final de estudos primários incluídos no MS, realizou-se então a extração dos dados. Nesta fase, as informações necessárias para responder às QPs foram extraídas e armazenadas em uma planilha para que pudessem ser analisadas e interpretadas posteriormente. Foram extraídos os seguintes dados: objetivos, abordagens do estudo; método do estudo (por exemplo, experimento controlado e/ou estudo de caso); como os dados foram coletados e analisados durante o estudo; os benefícios, conclusões e dificuldades encontradas, dentre outros.

\section{Resultados e Discussões}

A execução foi realizada conforme o planejamento descrito nas seções anteriores. A Figura 1 apresenta um resumo dos resultados da aplicação dos CI e CE em cada etapa. 
A busca automática encontrou 120 artigos em potencial, sendo que apenas 13 deles estavam de acordo com os critérios de inclusão e exclusão utilizados. Já a busca manual encontrou 65 artigos inicialmente, terminando em 21 artigos no final. A aplicação da técnica de backward snowballing resultou em 15 artigos, sendo 13 já encontrados no processo de busca (manual e automática) sendo assim descartados. No final, esse procedimento resultou em 2 novos artigos, finalizando em 36 artigos analisados neste mapeamento.

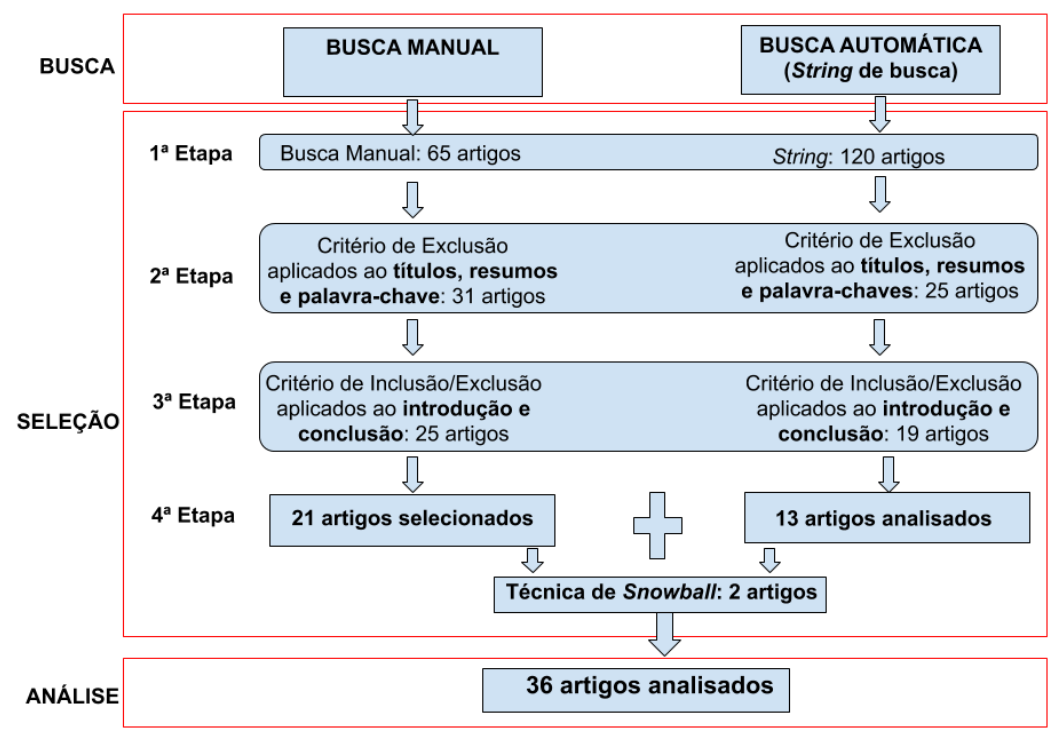

Figura 1: Estratégia de pesquisa e seleção.

As referências bibliográficas dos 36 artigos incluídos neste MSL podem ser visualizadas em: <encurtador.com.br/kzKLZ>. Nas seções seguintes, serão apresentadas, as respostas às questões de pesquisa, as limitações e ameaças do estudo e os trabalhos relacionados.

\subsection{Respostas as Questões de Pesquisa}

Nesta seção, apresentam-se as respostas às questões de pesquisa deste MSL. Um resumo das informações que foram extraídos de cada estudo incluído pode ser encontrado em: $<$ encurtador.com.br/vBDKN $>$.

\section{QP1: Quais as abordagens estão sendo propostas para o ensino de RC?}

Ao analisar os estudos selecionados, nota-se que as abordagens utilizadas para o ensino de RC são variadas, sendo classificadas nesse trabalho como: Ferramentas de Softwares, Metodologias, Jogos, Simuladores, Laboratórios Virtuais e Objetos de Aprendizagem.

As Ferramentas de Softwares pode ser vista como um software desenvolvido para realizar uma determinada tarefa educacional ou formação em um conjunto específico de habilidades. As Metodologias para o contexto do estudo são recursos que auxiliam no ensino de RC, tais como mapas conceituais, computação desplugada, sala de aula invertida, relatos de experiências, entre outros. Os Objetos de Aprendizagem são recursos digitais e não digitais que podem ser utilizados no processo de ensino e aprendizagem. Os Jogos podem ser digitais quando implementado em um meio computacional ou não digital, como jogos de tabuleiro e cartas. Já os Simuladores permitem aos estudantes a prática e a descoberta de conceitos fundamentais, além de estimular a busca de soluções de problemas na área de estudo. Por fim, os Laboratórios 
Virtuais são plataformas digitais com o intuito de suportar a realização de experiências práticas, tal como ocorre no contexto dos laboratórios reais.

Dentre os trabalhos incluídos, 7 são Ferramentas de Softwares, que corresponderam 19\% dos estudos. Três trabalhos [E9, E11 e E20] fazem o uso de ferramentas para auxiliar o processo de ensino e aprendizagem em RC, como destaca o estudo [E11], que desenvolveu uma ferramenta que permite a integração do Network Simulator (NS2) ao Moodle, proporcionando a criação e configuração de cenários de redes através de uma interface gráfica. Dois estudos [E22 e E27] desenvolveram Ambientes Virtuais Imersivos, um estudo [E35] utilizou ambientes virtualizados para ensinar RC e outro estudo [E25] desenvolveu um agente inteligente para auxiliar o aluno no processo de ensino e aprendizagem em RC em um ambiente virtual.

Também foi percebido um número relevante de artigos que abordaram o uso de Jogos para o processo de ensino e aprendizagem dos conteúdos de RC, sendo essa abordagem reportada em 7 estudos, correspondendo a 19\% do total. Destes, duas pesquisa [E2 e E7] aplicam jogos sérios, que podem ser definidos como uma atividade lúdica, pois é realizada em uma realidade simulada onde os participantes tentam alcançar, pelo menos, uma meta arbitrária, não trivial, agindo de acordo com as regras propostas pelo jogo (ADAMS, 2010). Outros quatro estudos [E1, E4, E5 e E8] foram reportados utilizando jogos digitais e [E3] fez uso de jogos não digitais.

É importante também destacar que 19\% dos estudos aplicaram Metodologias, o que demonstra que, embora os estudos empregadas em cada relato sejam distintos, eles podem ser combinados para se alcançar um objetivo de pesquisa maior. Os estudos [E18 e E19] utilizaram Computação Desplugada. Já [E23] faz uso de Realidade Aumentada, [E17] aborda o uso de Mapas Conceituais, [E21] utilizou Recursos Educacionais Abertos, [E29] Sala de Aula Invertida, enquanto o estudo [E15] aborda um relato de experiência no ensino de RC.

A utilização de Simuladores correspondeu a $17 \%$ e foi a abordagem utilizada em seis estudos [E10, E30, E31, E32, E34 e E36]. Cabe destacar o uso do simulador Packet Trace, como relata o estudo [E36]: "a utilização do Packet Tracer possibilita ao estudante observar e entender características relacionadas a comunicação de dispositivos que não são vistas no mundo real, melhorando o entendimento $e$ aprendizado."

Os Objetos de Aprendizagem foram utilizados em 14\% dos estudos, como destacado nas pesquisas [E6, E16, E24, E26 e E28]. Em contrapartida, os Laboratórios Virtuais foi a abordagem empregada em quatro estudo [E12, E13, E14 e E33], perfazendo um total de $12 \%$.

\section{QP2: Que conteúdos estão sendo abordados nos estudos?}

Houve uma variação nos conteúdos aos quais os estudos estavam sendo desenvolvidos. A maioria dos estudos apresentaram uma abordagem geral de $\mathrm{RC}$, perfazendo um total de nove estudos. Cinco estudos abordaram o conteúdo de Topologias. Quatro estudos tinham como propósito ensinar Segurança de Redes. Os conteúdos de Gerenciamento de Sistemas e Redes, Servidores, Roteamento IP e Instalação de RC, foram abordados em dois estudos respectivamente, perfazendo um total de 8 estudos. Os demais estudos apresentam um único conteúdo. A Tabela 2 apresenta os conteúdos abordados e as abordagens utilizadas em cada estudo.

Tabela 2: Conteúdos abordados e abordagens utilizadas.

\begin{tabular}{|c|c|c|c|c|c|c|}
\hline $\begin{array}{c}\text { Abordagens/ } \\
\text { Conteúdos }\end{array}$ & Ferramentas & Metodologias & Jogos & $\begin{array}{c}\text { Lab. } \\
\text { Virtuais }\end{array}$ & $\begin{array}{c}\text { Simuladore } \\
\text { s }\end{array}$ & OA \\
\hline V. $18 \mathrm{~N}^{\mathrm{o}} 1$, julho, 2020 & \multicolumn{6}{|c|}{ RENOTE } \\
\hline
\end{tabular}




\begin{tabular}{|c|c|c|c|c|c|c|}
\hline Visão Geral & $\begin{array}{l}\text { [E9, E11, } \\
\text { E25] }\end{array}$ & & [E5] & $\begin{array}{l}\text { E12, } \\
\text { E14] }\end{array}$ & E10 & $\begin{array}{l}{[\mathrm{E} 16,} \\
\text { E28] }\end{array}$ \\
\hline Falhas de Redes & & & [E1] & & & \\
\hline Segurança de Redes & [E22, E27] & & $\begin{array}{l}{[\mathrm{E} 2,} \\
\mathrm{E} 8]\end{array}$ & & & \\
\hline $\begin{array}{l}\text { Gerenciamento de } \\
\text { Sistemas e Redes }\end{array}$ & & & [E3] & & & [E24] \\
\hline Arquitetura TCP/IP & & & [E4] & & & \\
\hline Topologias & [E35] & {$[\mathrm{E} 17, \mathrm{E} 23]$} & & & {$[\mathrm{E} 32, \mathrm{E} 36]$} & \\
\hline $\begin{array}{c}\text { Servidores de Redes e } \\
\text { Servidor Linux }\end{array}$ & [E35] & & & & & [E6] \\
\hline Protocolo UDP & & [E15] & & & & \\
\hline Protocolo RIP & & & [E7] & & & \\
\hline Roteamento IP & & & & [E13] & & [E26] \\
\hline Modelo OSI & & [E18] & & & & \\
\hline Firewall & & [E19] & & & & \\
\hline $\begin{array}{c}\text { Fragmentação IPV4, } \\
\text { IPV6 }\end{array}$ & [E20] & & & & & \\
\hline IPV6 & & [E21] & & & & \\
\hline Administração de Redes & & E29] & & & & \\
\hline Instalação de RC & & & & & {$[\mathrm{E} 30, \mathrm{E} 31]$} & \\
\hline Infraestrutura de Redes & & & & [E33] & & \\
\hline $\begin{array}{l}\text { Geração de tráfego e } \\
\text { Monitoramento de } \\
\text { pacotes }\end{array}$ & & & & & [E34] & \\
\hline
\end{tabular}

QP3. Quais concepções e/ou teorias pedagógicas estão sendo utilizadas nos estudos?

Esta questão tem como objetivo apontar as principais concepções e/ou teorias pedagógicas utilizadas nos estudos. As abordagens que foram citadas são apresentadas na Tabela 3.

Tabela 3: Abordagens pedagógicas apresentadas nos estudos.

\begin{tabular}{|c|l|c|}
\hline $\begin{array}{c}\text { Abordagens } \\
\text { Pedagógicas }\end{array}$ & \multicolumn{1}{|c|}{ Descrição } & Estudos \\
\hline $\begin{array}{c}\text { Aprendizagem } \\
\text { Significativa }\end{array}$ & $\begin{array}{l}\text { Identifica o favorecimento da aprendizagem com a identificação das relações } \\
\text { entre os conceitos (AUSEBEL, 1976). }\end{array}$ & [E2 e \\
E17]
\end{tabular}

A análise dos resultados mostra que $86 \%$ das pesquisas (31 artigos) não se preocuparam em ter ou pelo menos citar a adoção de uma teoria de aprendizagem que fundamente o desenvolvimento e a aplicação dos estudos. Este é um ponto que precisa ser revisto pela comunidade, uma vez que a ausência de uma teoria de aprendizagem na fundamentação de uma abordagem pode interferir diretamente no sucesso do processo de ensino e aprendizagem proposto por ela.

\section{QP4: Como está sendo realizada a avaliação dos estudos?}

Para Silva et. al., (2018) a avaliação é um elemento importante para o ensino de qualquer área do conhecimento. Nesse contexto, o objetivo dessa questão é responder os seguintes questionamentos: Que tipos de estudos estão sendo realizados pelos pesquisadores? Qual a técnica de coleta e análise de dados utilizada no estudo?

Com relação aos tipos de estudos, foram encontrados apenas um tipo, que foi o Estudo de Caso. Os estudos de caso visam observar um atributo específico e estabelecer o relacionamento entre atributos diferentes (TRAVASSOS, GUROV e AMARAL, V. $18 \mathrm{~N}^{\mathrm{o}} 1$, julho, 2020 RENOTE DOI: 
2002), sendo utilizado para monitorar a utilização a aplicabilidade das abordagens durante o estudo. Dos trabalhos encontrados por este MSL, 8 realizaram estudos de caso, correspondendo a $22 \%$ do total. Em 4 estudos os autores descreveram que utilizaram Experimento, mais não deixa claro se é um experimento controlado ou quasiexperimento, no qual nem todas as características de aleatorização ou de controle estão presentes.

Em relação à técnica de coleta e análise de dados, a maioria dos estudos (16 artigos $-80 \%$ do total) utilizaram questionários como a técnica principal para coleta de dados. Nestes casos, foram empregadas perguntas para se avaliar a opinião dos alunos em relação a um conjunto de aspectos, como satisfação, motivação e aprendizagem. Outra técnica utilizada foi a de observação, empregada para avaliar o comportamento dos estudantes no momento da aplicação das tecnologias investigadas. Esta técnica está presente em $10 \%$ do total ( 2 estudos). Por fim, 2 trabalhos (10\%) relatam a utilização de entrevistas para se obter feedback após a aplicação dos estudos.

Quanto à esta questão, muitos trabalhos (28 dos 36) não apresentam uma avaliação adequada das abordagens propostas. Em sua maioria, os resultados são descritos apenas informal e subjetivamente, por exemplo, “... após o uso da abordagem proposta pode-se constatar uma melhora no desempenho/aprendizado dos alunos". Outros estudos reportam que fizeram um experimento mais não deixa claro se foi um experimento controlado, quasi-experimento ou estudo de caso.

Com base neste cenário acima descrito, pode-se notar que há necessidade de que a comunidade científica reúna esforços no que tange à elaboração de trabalhos que visem a avaliar as abordagens propostas de modo mais efetivo, por meio de estudos de caso, experimentos controlados, quasi-experimentos, entre outros. Isso é imprescindível para que evidências a respeito da real eficácia desses estudos possam ser levantadas e conhecidas pela comunidade.

\section{QP5: Os estudos desenvolvidos suportam algum tipo de Tecnologia Assistiva?}

Dos 36 estudos analisados, apenas dois estudos [E24 e E26] apresentavam suporte para pessoas com algum tipo de necessidade especial. Por exemplo, o [E26] apresentar os resultados da inclusão de acessibilidade audiovisual em animações disponíveis em um repositório on-line referente a disciplina de $\mathrm{RC}$, possibilitando a utilização dessas animações por deficientes visuais e permitindo uma maior igualitariedade no processo de ensino e aprendizagem. Os dois estudos trabalham a acessibilidade audiovisual.

Desta forma, estes dados revelam a necessidade de mais estudos que possam incluir alunos com algum tipo de necessidade especial. Em outras pesquisas, como, Silveira Júnior et. al. (2019), Silva, Medeiros e Aranha (2014) e Silva et. al. (2018) não foram discutidos estudos que tratavam algum tipo de suporte quanto as tecnologias assistivas.

\subsection{Ameaças a validade do MSL}

No geral, as principais ameaças à validade da RSL são em relação ao viés de seleção de publicações e possíveis imprecisões na extração dos dados dos artigos, sendo as formas de controle utilizadas e potenciais ameaças existentes discutidas a seguir. As pesquisas foram concebidas empregando recursos eletrônicos e manuais. Estes foram pesquisados após a implementação de pesquisas experimentais, strings de busca e utilização de dicionários de sinônimos. Apesar disso, não é possível garantir que todos os estudos relevantes foram coletados e há um pequeno risco de que alguns estudos podem ter sido omitidos, devido aos termos de busca utilizados. 
O processo de extração de dados também pode resultar em dados irrelevantes. Isto pode ocorrer, porque o processo de extração de dados foi realizado somente por um pesquisador. O desenvolvimento de um protocolo do MSL e o uso de uma estratégia de verificação da qualidade da revisão ajuda a garantir que este não foi o caso. Finalmente, é possível que os critérios de inclusão possam, ocasionalmente, terem excluídos alguns estudos relevantes. Isso pode ocorrer porque os critérios implementados nos estudos não continham palavras-chaves apresentadas nos títulos ou nos resumos. Esse risco foi minimizado através da não remoção dos artigos que gerassem dúvidas.

\subsection{Trabalhos Relacionados}

Silveira Júnior et. al., (2019) realizaram um estudo sobre o desenvolvimento e a utilização de jogos e simuladores no ensino de RC, que resultou no total de 27 artigos. Para tal utilizaram uma metodologia de MSL visando extrair, catalogar, analisar e sintetizar dados de artigos publicados nos últimos 5 anos (2014-2019) através de uma string de busca. Silva et. al. (2018) realizaram uma RSL analisando o cenário nacional no uso de jogos voltados para o ensino e aprendizagem de Computação, dos 145 estudos encontrados, somente 3 artigos eram jogos para o ensino de RC.

Este estudo desenvolveu um processo similar ao apresentado por Silveira Júnior et. al., (2019), aplicando a técnica de pesquisa secundária (o MSL) para realizar uma análise abrangente e sistemática da utilização de tecnologias educacionais no processo de ensino e aprendizagem em RC no cenário nacional. Em contrapartida a diferença para o estudo de Silveira Júnior et. al. (2019) é que além de uma string de busca, realizamos uma pesquisa manual e aplicamos a técnica de backward snowballing. Além disso, incluímos não somente jogos e simuladores, mais ferramentas, objetos de aprendizagem, laboratórios virtuais para aumentar o escopo do estudo.

\section{Considerações Finais}

Nesta pesquisa, apresentamos os resultados de um MSL sobre o uso de tecnologias educacionais para auxiliar o processo de ensino e aprendizagem em RC publicados nos últimos cinco anos, em relevantes eventos e periódico na área no cenário nacional. A busca realizada neste mapeamento resultou na pré-seleção de 185 estudos, dentre os quais 36 foram incluídos para a extração de dados. Os estudos para o ensino e aprendizagem de RC estão voltados para melhorar o rendimento dos alunos, atrair o interesse dos alunos e auxiliar os professores em cenários diversos de aprendizagem, por exemplo, a criação de cenários de redes o mais prático possível, além da possibilidade de adicionar e gerenciar, roteadores e máquinas virtuais através de um laboratório virtual.

Cinco QPs foram criadas com o objetivo de verificar o cenário nacional da aplicação de tecnologias educacionais no ensino de RC. Vários resultados puderam ser extraídos dos dados recolhidos dos estudos. Com eles, pudemos observar que (i) Ferramentas e Jogos são as abordagens que foram mais frequentemente usadas nas pesquisas; (ii) os estudos estão sendo mais aplicado no ensino superior; (iii) não há um foco específico em um conteúdo para ensino de RC, pois encontramos conteúdos diversos; (iv) a falta de avaliação e aplicação dos estudos em cenários reais é um problema destacado nas pesquisas; (v) falta de evidências científicas que comprovem quais estudos são capazes de gerar resultados para a aprendizagem.

Os estudos primários analisados são uma importante fonte de informações sobre as diversas mudanças enfrentadas nessa área ao longo dos anos. Uma das observações mais importantes percebidas foi a baixa aplicação de métodos empíricos como meio de 
avaliação dos resultados obtidos pelas pesquisas desenvolvidas, poucos foram os estudos que apresentaram algum resultado. Destacamos também a falta de fundamentação pedagógica nos estudos. Os estudos reportam que em $78 \%$ não foram aplicados nenhum método empírico de pesquisa. Com base neste cenário, pode-se notar que há necessidade de que a comunidade científica nacional reúna esforços no que tange à elaboração de trabalhos que visem a avaliar as abordagens propostas de modo mais efetivo, por meio de estudos que apliquem algum método de pesquisa e apresente resultados. Apesar dos resultados apresentados serem considerados ainda incipientes.

As publicações classificadas aqui como não empíricas representam as pesquisas de caráter fundamentalmente descritivo, como a apresentação de relato de experiência, entrevistas, oficinas e aplicação de questionários.

\section{Referências}

Adams. E. Fundamentals of game design. In: New Riders, 2nd edition. 2010.

Ausubel, D. P. Psicología educativa: un punto de vista cognoscitivo. Trad. Roberto Helier Domínguez. Trillas: México, 1976.

Bertoncelli Junior, C. A.; Aikes Junior, J.; Lopes, R. P. Desenvolvimento e Aplicação de um Jogo na Disciplina de Gerenciamento de Sistemas e Redes. In: Simpósio Brasileiro de Jogos e Entretenimento Digital, p.1321-1324, 2019.

Costa, V. R.; Viana, W.; Coutinho, E. F. Um Relato sobre a Monitoria da Disciplina de Redes de Computadores no Curso de Sistemas e Mídias Digitais. In: Revista Sistemas e Mídias Digitais, p.1-12, 2017.

Ferreira, K. H. A.; Lima, R. W.; Lima1, M. V. A.; Chaves, J. O. M.; Silva, T. R. Integrando o Network Simulator 2.0 a um Ambiente Virtual de Aprendizagem. In: XXII Workshop sobre Educação em Computação, 1613-1622, 2014.

Hassan, E. B. Laboratório Virtual 3D para ensino de Redes de Computadores. In: XIV Simpósio Brasileiro de Informática na Educação, Rio de Janeiro - RJ, 2003.

Hmelo-Silver, C. E; Barrows, H. S. Goals and strategies of a problem-based learning facilitator. In: Interdisciplinary Journal of Problem-based Learning, 1(1):4, 2006.

Jalali, S.; Wohlin, C. Systematic literature studies: Database searches vs. backward snowballing. In: IEEE International Symposium on Empirical Software Engineering and Measurement, p.29-38, New York, NY, USA, 2012.

Kitchenham, B.; Brereton, P.; Budgen, D.; Turner, M.; Bailey, J.; Linkman, S. Systematic literature reviews in software engineering - A systematic literature review. In: Information and Software Technology, v.51, p.7 -15, 2009.

Silva, T. R.; Cordeiro, J. R.; Santos, R. S. F.; Santos, F. G.; Aranha, E. H. S.; Silva, F. G. Uma Análise do Cenário Nacional do Uso de Jogos para o Ensino e Aprendizagem de Computação. In: Simpósio Brasileiro de Jogos e Entretenimento Digital, 2018.

Silveira Júnior, G.; Medina, R. D.; Lima, P. R. B. D.; Rocha M. A. E. Mapeamento Sistemático sobre o desenvolvimento e a utilização de Jogos e Simuladores no ensino de Redes de Computadores. In: Revista Novas Tecnologias na Educação, 2019.

Silva, T. R.; Medeiros, T. J.; Aranha, E. H. D. S. Jogos digitais para ensino e aprendizagem de programação: uma revisão sistemática da literatura. In: Simpósio Brasileiro de Informática na Educação, p. 692-701, 2014.

Travassos, G., Gurov, D., Amaral, E. Introdução à Engenharia de Software Experimental. In: Relatório Técnico ES-590/02-abril, Programa de Engenharia de Sistemas e Computação, COPPE/UFRJ, 2002.

Yahya, S.; Ahmad, E. A.; Jalil, K. A.; Mara, U. T. The definition and characteristics of ubiquitous learning: A discussion. In: International Journal of Education and Development using Information and Communication Technology, v.6, p.1-12, 2010. 